\title{
A cost-effective architecture for optical multistage interconnection network.
}

\begin{abstract}
In this paper a new architecture for Optical Multistage Interconnection Networks (OMINs) has been proposed to avoid crosstalk problem. At the same time, the probablity of losing pass through an optical long connection path is reduced in this architecture. The new architecture is inherent form the standard OMIN by converting two switches of the network to one switch in each row. By reducing the number of switches in new architecture, the reduction in the execution time is considered. The modifying in the number of passes via the same low stage transformation is negligible. The ability of the new architecture to decrease cost and avoid crosstalk has been validated through simulations that show improvement in the network performance in terms of approximately $30 \%$ reduction in the execution time.
\end{abstract}

Keyword: Omega networks; Switch element; Low stage network; Crosstalk; Bandwidth. 